评 述

\title{
细菌泳动时的细胞取向：理论模拟与实验测定的 研究进展
}

\author{
平立岩
}

Max Planck Institute for Chemical Ecology, Beutenberg Campus, Jena 07745, Germany

E-mail: lping@ice.mpg.de

收稿日期: 2011-10-12; 接受日期: 2012-03-03

马克・普朗克学会资助项目

\begin{abstract}
摘要 小型细菌的运动通常由两部分组成：随细菌的种类不同，近似直线的前进态会被不 断出现的停顿、后退或者转向所间隔. 端生单鞭毛细菌的鞭毛是生长在由父母代遗传的那个 细胞极(称为老细胞极), 细菌的化合物感应受体聚集在鞭毛的周围. 向前游泳时, 处于前面 的永远是在上一次细胞分裂过程中刚刚产生的那个细胞极(称为新细胞极). 而具有周生鞭毛 的大肠杆菌游动时, 其鞭毛常在老细胞极的后面形成束. 大肠杆菌前进态的细胞取向与端生 单鞭毛细菌有一定的相似性. 在接近一个固相表面时, 周生鞭毛细菌会循一个圆形的轨迹前 进，此时的前导细胞极会微微向下倾斜。一些端生单鞭毛细菌在固相界面附近也会作圆周运 动，可是却不在前进时，而是在后退时；此时的前导极又是朝向上方的. 目前，对于细菌在液 体一空气界面处的行为研究几乎空白. 现有的生物物理学理论模型可以解释一部分现象, 但更多的内部规律还有待进一步阐明. 生物物理学理论和分子生物学技术的结合必将大大推 动人们对细菌运动规律的了解.
\end{abstract}

关键词

细菌运动 细胞取向 流体动力学 布朗运动 壁效应
越来越多的实验证据显示, 细菌能够主动地 对外界刺激, 如光、化合物和氧气等作出反应 ${ }^{[1]}$. 它 们沿着化合物浓度梯度的方向进行迁移的行为被称 为趋化现象 (chemotaxis). 目前, 对趋化现象的了解 已经比较透彻. 关于细菌在趋化现象中的迁移策略 和信号传导机制, 已有不少详尽的综述讨论 ${ }^{[1-4]}$. 然 而, 运动本身很可能是一个先于趋化现象而产生的 进化事件 ${ }^{[5]}$. 通过对趋化现象的研究, 已经对细菌的 运动机理有了初步了解, 但对某些泳动机理的问题, 比如细胞体在泳动中的取向, 了解还相对有限. 本文 旨在将对于这个问题的实验研究和理论探讨的成果
系统化.

微生物机体相对于周围环境介质的相对运动从 属于物理学中力学的一个分支一一流体动力学(hydrodynamics). 细菌的细胞体长度通常约 $2 \mu \mathrm{m}$ 左 右. 这样的微小物体前进时只能依靠黏性切变力 (viscous shear), 而人们所熟悉的肉眼可见大型生物 体的运动则依赖惯性阻力(inertial resistance), 其机理 完全不同. 关于细菌运动的物理学模型沿革, 可以参 阅 Ramia 等人 ${ }^{[6]}$ 的论文, 以及其中引用的一些文献. 很多细菌是通过一根或者一束大约 $10 \mu \mathrm{m}$ 长的鞭毛 旋转产生的动力前进的. 在黏性介质中, 一个物体加 
速所需的惯性力 (inertial forces)和产生切变的黏性力 (viscous forces)的比值, 被定义为雷诺指数 (Reynolds number). 当大肠杆菌在水中全速前进时(约 $25 \mu \mathrm{m} / \mathrm{s}$ ), 其运动的雷诺指数约为 $1 \times 10^{-5[1]}$, 即此时惯性力的作 用可以忽略不计.

小型细菌的运动不免会受到布朗运动(Brownian motion)的干扰 ${ }^{[7]}$. 布朗运动指微小物体的随机热运 动. 因此, 小型细菌无法完成一个延续长达数秒的直 线运动, 布朗运动会不断将其推向新的方 向 $^{[3]}$. 随着 细胞体积的增大, 布朗运动的影响逐渐减弱 ${ }^{[8]}$. 在 $32^{\circ} \mathrm{C}$ 下, 平移扩散和旋转扩散能使大肠杆菌的前进 轨迹在 $1 \mathrm{~s}$ 内相对初始方向平均偏离 27 度 ${ }^{[9]}$. 类似情 况在有端生丛鞭毛的小着色菌(Chromatium minus)上 也有发生, 只不过由于它比大肠杆菌要大近 20 倍 ${ }^{[10]}$, 所以偏离程度要小一些. 旋转布朗扩散的程度总是 与物体大小的立方成反比 ${ }^{[11]}$. 小着色菌在 $23^{\circ} \mathrm{C}$ 下, $1 \mathrm{~s}$ 内偏离大约 8.4 度 $^{[12]}$.

为了能够不断校正前进方向, 周生鞭毛细菌的 泳动由两个交替发生的步骤组成: 一个是持续较长 时间(约 $1 \mathrm{~s}$ ) 的近似直线前进(run); 另一个是相对较 快(约 $0.1 \mathrm{~s}$ )的转向 (tumble)(图 1). 它们的鞭毛在

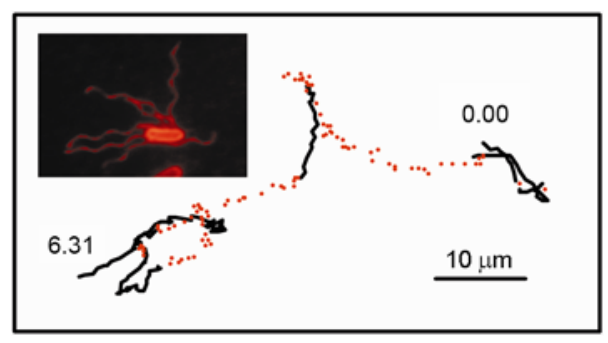

图 1 一个大肠杆菌 AW405 菌株在 LB 培养基中的自由 泳动轨迹

视频由高速摄像机以 $25 \mathrm{~ms} /$ 张速度拍摄. 细菌向前游泳速度约 32 $\mu \mathrm{m} / \mathrm{s}$. 红色点显示细菌游出聚焦平面后的可能位置. 本图的实时视 频详见网络版附录文件视频 S1. 欺入图为一个经过荧光染色后的 大肠杆菌 AW405 菌株的照片, 图中着重显示其螺旋状的周生鞭毛
前进时形成一个左手螺旋的束. 假设在细菌的后面 进行观察, 就会看到鞭毛束沿着逆时针方向以大约 $100 \mathrm{~Hz}$ 速度旋转, 而细胞体则以大约 $10 \mathrm{~Hz}$ 的速度沿 顺时针方向旋转 ${ }^{[13]}$. 如果其中有些鞭毛的旋转方向 发生改变, 这些鞭毛会由左手螺旋转换为右手螺旋. 这时, 或者整个鞭毛束突然散开 ${ }^{[14]}$, 或者一根或几根 鞭毛从鞭毛束中分离出来 ${ }^{[13]}$. 散开鞭毛产生的不平 衡推力(thrust), 造成细菌改变泳动方向.

在水中生活的端生单鞭毛细菌不会转向, 而且 它们游泳的速度要比周生鞭毛细菌快得多(表 1). 有 些单鞭毛细菌的鞭毛是右手螺旋的, 有些是左手螺 旋的. 右手螺旋的鞭毛在推动细菌前进时沿顺时针 方向旋转; 左手螺旋鞭毛推动细菌时沿逆时针方向 旋转. 当这些细菌的鞭毛倒转时, 细菌就向后退 (back-up). 后退在功能上等同于周生鞭毛细菌的转 向, 只不过端生单鞭毛可能在旋转方向改变时并不 改变螺旋形态 ${ }^{[15]}$. 假设没有其他外加因素, 后退的细 菌会沿着它们前进时的同一路径退回. 然而, 布朗运 动使小型的单鞭毛细菌的泳动轨迹成“之”字形, 因 为它不但改变了它们的前进轨迹, 而且更加严重地 改变了它们后退时的细胞取向 ${ }^{[15,23]}$. 最近的研究显 示, 溶藻弧菌(Vibrio alginolyticus)还能在后退和下一 次前进态之间闪动它们的单鞭毛, 从而使细胞体的 朝向更随机 ${ }^{[26]}$. 已有假说认为, 后退游泳策略是由对 高剪切流液体环境的适应进化产生的, 因为转换角 度较小时, 剪切流的影响减低 ${ }^{[27]}$. 鞭毛闪动行为的引 入似乎是趋化作用从静态液体环境向流动环境转变 时的一种无奈选择.

有光和能力的球形红细菌(Rhodobacter sphaeroides) 也比大肠杆菌游动快, 其平均速度约 $35 \mu \mathrm{m} / \mathrm{s}$. 它唯 一的一根鞭毛处于细胞体的赤道区. 这根鞭毛静止 时是一个右手螺旋的扁平线圈, 当它沿顺时针方向 旋转时会伸展, 从而推动细菌沿细胞体短轴方向平

\section{表 1 文献中一些端生单鞭毛细菌的游泳行为}

\begin{tabular}{|c|c|c|c|c|c|c|c|c|c|}
\hline \multirow{2}{*}{ 种类 } & \multicolumn{2}{|c|}{ 细胞体 } & \multicolumn{2}{|c|}{ 鞭毛 } & \multicolumn{2}{|c|}{ 自由泳动速度 $(\mu \mathrm{m} / \mathrm{s})$} & \multicolumn{2}{|c|}{ 近界泳动轨迹 } & \multirow{2}{*}{ 参考文献 } \\
\hline & 形状 & 弯曲 & 螺旋 & 旋转 a) & 前进 & 后退 & 前进 & 后退 & \\
\hline V. alginolyticus ${ }^{\text {b) }}$ & 弧菌 & 微曲 & 左手 & 逆时针 & 37.3 & 52.9 & 微曲 & 逆时针 & [15 18] \\
\hline C. crescentus $^{\mathrm{c})}$ & 近弧菌 & 左旋 & 右手 & 顺时针 & 41.7 & 同前 & 未知 & 顺时针 & [19 22] \\
\hline P. citronellolis & 杆状 & 未知 & 左手 d) & 逆时针 & $55.8^{\mathrm{e})}$ & 稍慢 & 顺时针 d) & 未知 & [23 25] \\
\hline
\end{tabular}

a) 从后部观察向前游泳的细菌; b) 数据源于一个没有周生鞭毛的突变体; c) 数据源于一个没有附着纤毛的突变体; d) 推论结果, 详见 正文; e) 参考数据源于对 P. aeruginosa 的测量, P. citronellolis 的参数未见报道 
移. 一旦旋转中止, 鞭毛会重新收缩到细胞体旁, 从 而使布朗运动对细胞朝向的改变更有效 ${ }^{[28]}$. 在这个 收缩过程中, 鞭毛圈还会缓慢地转动, 应该对细胞体 取向随机化也有促进作用 ${ }^{[29]}$.

无论细菌采用哪一种游泳模式, 它们的轨迹都 是随机位移(random walk) ${ }^{[1,23,28]}$. 通过调整前进态的 持续时间, 细菌能够获得一个向有利方向的净位移, 即当它们向适宜方向泳动时, 前进态的时间就延长; 当向不适方向泳动时，前进态的时间就缩短. Vladimirov 等人 ${ }^{[30]}$ 的研究显示, 通过控制倒转鞭毛的 数量, 周生鞭毛细菌向适宜方向泳动时的转向角度 与不适方向相比平均降低 3 度左右. 这也大大促进了 它们向有利方向迁移.

\section{1 前进态的细胞朝向}

根据 Berg 和 Purcell ${ }^{[31]}$ 的经典论文中提出的生 物物理学模型, 在一个理想化的球形细胞表面, 如 果 $0.1 \%$ 的面积上均匀分布着化感受体, 就能最大效 率地捕捉周围介质中的信号分子. 然而, 后来的研 究 ${ }^{[32,33]}$ 显示, 细胞受体是成族存在的. 在新月柄杆菌 (Caulobacter crescentus)的扩散胞(swarmer cell)上受 体聚集在有鞭毛的细胞极 ${ }^{[32]}$; 在大肠杆菌中, 受体 则分别聚集在两个细胞极上 ${ }^{[33]}$. 细菌的两个细胞极 中的一个是从上代细胞继承的, 称为老细胞极 (old pole), 而另一个则是在刚刚发生的细胞分裂过程中 由赤道板形成的, 称为新细胞极 (new pole). 化感受 体在细胞极上随时间线性积累 ${ }^{[34]}$. 一个新细胞极只 在随后的一个细胞周期中存在; 在此后的细胞分裂 过程中, 这同一个细胞极(此时已是老细胞极)上的丝 氨酸受体 Tsr 的数量以每个细胞周期相当于新细胞极 $(83 \pm 4) \%$ 的速度增长. 到大约 3.5 个分裂周期后, 细胞 极上的受体数量趋于饱和 ${ }^{[34]}$. 在一个指数生长的随 机细菌群体中, 老细胞极上的 Tsr 受体数量平均为新 细胞极的 2.6 倍. 不过, 小化感受体簇, 甚至单独的 受体蛋白也遍布细胞表面 ${ }^{[34 ~ 36]}$. 有的学者认为, 这 些小族或单独受体可以保证传递到细胞内的信号更 均匀分布; 另一些则认为, 这是为了确保后代细胞在 分裂后及时获得受体分子 ${ }^{[35]}$.

化感受体在细胞极形成簇似乎显得没有必要, 而且对它们聚集的机制目前还没有解释 ${ }^{[4,37]}$. 一个典 型的大肠杆菌细胞极上往往存在超过 7500 个受体双
聚体 ${ }^{[37]}$, 进行有效的信号传导只要 3 个双聚体在一起 就足够了 ${ }^{[38]}$. 像大肠杆菌一样的小型细菌无法测量 细胞两极存在的空间浓度梯度差异. 要使一个理想 的球形细菌的前后两端捕捉到的信号浓度提高 1 倍, 那么细菌的游动速度必须提高 100 倍 ${ }^{[31]}$.

化感作用实际是通过比较当时的浓度与几秒钟 之前感受的浓度的差别完成的 ${ }^{[39]}$. 计算机模拟结果 显示，受体分子间的异构相互作用(allosteric interaction) 可以大大提高信号转导效率 ${ }^{[40,41]}$, 但一个功能簇中 的最佳分子数量又超乎想象的小，不同的数学模型 预期大约在 5 25 个受体双聚体之间 ${ }^{[42 ~ 44]}$. 若真像 Greenfield 等人 ${ }^{[36]}$ 提出的随机成核膜型 (stochastic nucleation mode)显示的那样，细胞极上的巨大受体 簇只不过是一种无法避免的累赘, 那么这些簇中的 受体是否具有功能就不那么重要了. 但假如说那些 老的受体分子是有活性的, 那么老细胞极会不会比 新细胞极对信号更敏感呢? 这个问题似乎只能通过 实验去回答：遗传学和分子生物学的手段允许人为 地重组受体分子, 并通过引入阻碍基团关闭其活性 中心. 通过一个活性可诱导的蛋白酶可以控制阻碍 基团的存在与否. 比较蛋白酶基因诱导前后的细胞 反应就可以比较新合成受体和老的继承受体的功能 性问题.

在游泳细菌的转向过程中, 周生鞭毛细菌的两 个细胞极可以不断交换位置 ${ }^{[45]}$. 可是, 处于指数生长 期的大肠杆菌群体中，大约 $75 \%$ 的自由泳动细菌的 老细胞极是拖在后面的. 这是由于在靠近老细胞极 的细胞表面存在不对称分布的鞭毛的结果 ${ }^{[46]}$. 在转 向的过程中, 鞭毛的旋转方向逆转, 多出来的不对称 鞭毛使得细胞体更趋向于选择新细胞极位于前端的 朝向. 这个假说和以前发表的观测结果一致, 大肠杆 菌在转向后的角度改变平均值为 68 度 ${ }^{[7]}$. 如果是完 全随机的话，这个值就会是 90 度.

在周生鞭毛细菌上, 多出来的不对称鞭毛处在 距离老细胞极较近的位置, 即不对称鞭毛靠近那个 较大的受体簇. 这在一定意义上和端生单鞭毛细菌 相似. 端生单鞭毛细菌的鞭毛和受体簇都处于老细 胞极 ${ }^{[32]}$. 如果单纯考虑鞭毛束和受体簇在泳动细菌 上的相对位置, 周生鞭毛细菌则呈现一个介于端生 单鞭毛的柄杆菌和单鞭毛位于中央的红细菌之间的 连续变化分布(图 2A). 尽管球形红细菌的细胞极受 体簇是与大肠杆菌相似的不对称分布 ${ }^{[47]}$, 当细菌 


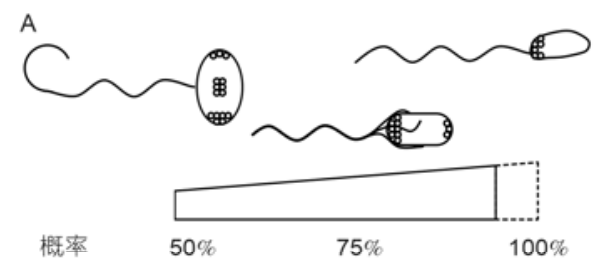

B

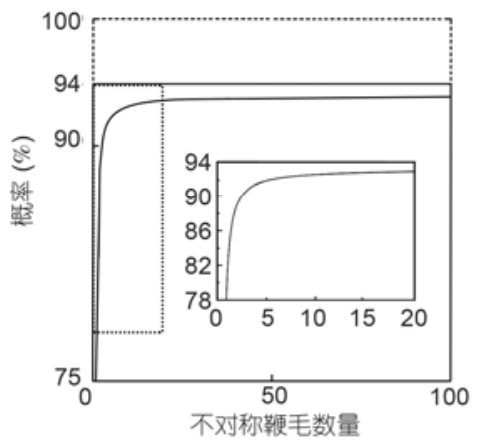

图 2 细菌向前游泳时老细胞极趋后的概率

这个概率同时显示了细菌中感应受体和鞭毛运动耦合的潜在效率. 虚线框为周生鞭毛细菌理论上无法达到的区域. A: 不同形态细菌 的概率. 中生鞭毛的球形红细菌(左)人为设定为 $50 \%$; 端生鞭毛的新 月柄杆菌扩散胞(右)设定为 100\%; 大肠杆菌(中)则平均为 $75 \%$, 且依 据每个细胞上的不对称鞭毛数量和位置而连续变化. 图中小圆环表 征细菌的化感受体. 尽管球形红细菌中还存在位于细胞中央的细胞 质受体群，但这对 50\%概率的设定没有影响; B: 周生鞭毛细菌老细 胞极趋后概率和不对称鞭毛数量的关系. 嵌入图为点线框所围区

体沿短轴方向平移时, 它们和鞭毛的相对距离相等. 一种双端鞭毛的海洋弧菌泳动时也是沿细胞的短轴 平移 ${ }^{[48]}$. 它的两个细胞极上的氧分子受体区各自独 立控制邻近鞭毛束的转动以调整泳动方向. 可惜还 没有人研究化感受体簇的位置以及大小与细胞极年 龄的关系.

在低雷诺指数条件下, 一个由数根鞭毛形成的 束所产生的扭矩和单根鞭毛几乎相同 ${ }^{[13]}$, 但如果考 虑推进器与检测器的相对位置, 不对称鞭毛数量多 的周生鞭毛细菌的泳动行为则更接近端生单鞭毛细 菌(图 2B). 多出来的不对称鞭毛距离细胞极的大受 体簇越近, 则受体与鞭毛耦合越紧密 ${ }^{[46]}$. 这正是人们 对端生单鞭毛的新月柄杆菌扩散胞中受体簇处于鞭 毛基座附近 ${ }^{[32]}$ 给出的解释 ${ }^{[45]}$. 由于旧细胞极上的大 受体簇与不对称鞭毛的距离更近, 它引发不对称鞭毛 逆转的效率比新细胞极上的受体簇要高的多. 实验 显示, 具有多个不对称鞭毛的大肠杆菌细胞在存在 化合物浓度梯度时迁移的效率比其他细胞高得多 ${ }^{[46]}$.

\section{2 固相界面附近的头朝下运动}

能动细菌不可避免地会接触到介质界面, 比如, 水传播病原在侵入寄主组织时; 浮游态水生细菌开 始固着生长时; 海洋细菌流连在释放营养物质的藻 类表面时; 或者根际细菌入驻植物根系时等. 在靠近 界面时, 细菌的游泳行为与在自由介质中相比有很 大变化. 由一个邻近的固相界面所产生的物理影响 称为壁效应(wall effect).

当大肠杆菌在层流介质中泳动时, 如果剪切流 的强度不会阻止正常泳动, 它们就会采取细胞长轴 和液流相一致的方向, 同时前导细胞极微微向下倾 斜 ${ }^{[49]}$. 细胞朝向液流的方向是剪切流存在的必然结 果 ${ }^{[49]}$. 没有剪切流时, 细菌会作圆周运动(图 3A). 不 过, 头朝下的姿势却并不需要剪切流的影响 ${ }^{[50]}$.

流体动力学计算显示, 一个非圆形细胞(无鞭毛 长粗圆)在一个非滑移的固相界面附近运动时, 该细胞 会受到来自界面的和迎面液流的双重曳力的作用 ${ }^{[51]}$. 由界面产生的曳力在细胞表面不同区域的差异作用 效果会使细胞倾向于向下倾斜; 而来自于迎面液流 的曳力(其实也是壁效应产生的)则使细胞倾向于保 持和界面平行. 由此产生的两个相互竞争的扭矩会 使细胞保持一个相对于界面的特定倾角. 于是, 细菌 采取头朝下的姿势(图 3B). 这个倾斜的角度与细菌 与界面间的距离和细胞长度与直径的比呈现一定的 相关性. 在一个更为全面的数学模型中, 当不同的细 胞几何形状以及鞭毛特征都被考虑进来, 细菌的细 胞形态在决定近壁泳动行为中显得至关重要 ${ }^{[52]}$. 有 的细胞形态会使细菌碰撞并离开界面, 而另一些细 胞则不断靠近，并最终保持在距界面一定高度处.

根据这些研究，一个没有鞭毛的球形细胞会由 于壁作用而向前滚动 ${ }^{[50]}$; 但如果有鞭毛存在, 滚动 就不会发生 ${ }^{[52]}$. 这个假说可以通过研究自然存在的 球菌, 如周生鞭毛的漫游球菌 (Vagococcus) 或人为 制造的球菌加以验证. 已知通过人为敲除细胞骨架 蛋白 $\mathrm{MreB}$ 和其他细胞形态相关蛋白, 可以产生球 形细胞 ${ }^{[3,54]}$. 由于这样的细胞一般比较脆弱, 所以实 验要在等渗溶液中进行. 还有一类比较有趣的球菌 是具有两束丛生鞭毛的趋磁球菌 MC-1 菌株. 它的两 个鞭毛束位于细胞的同一个半球 ${ }^{[55]}$. 这两个鞭毛束 如何协调运动来驱动球菌的平移是另一个值得研究 的问题. 


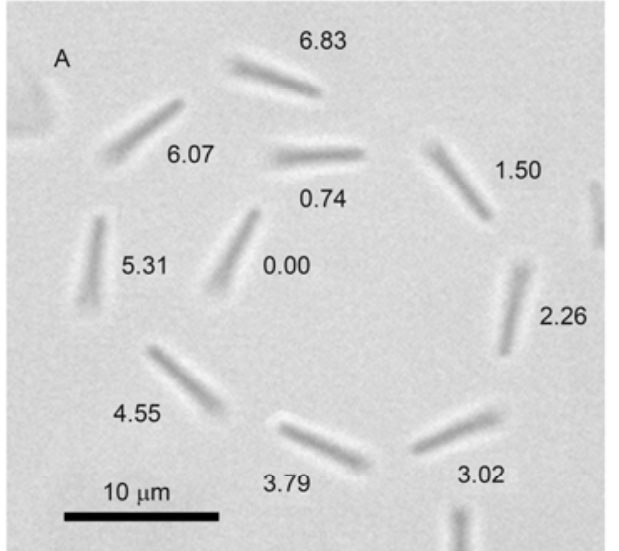

B

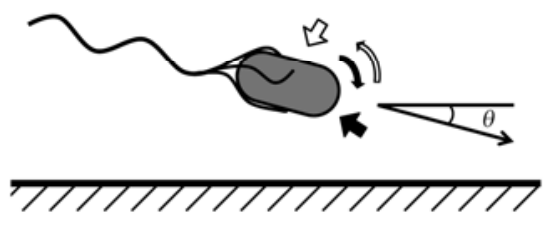

c

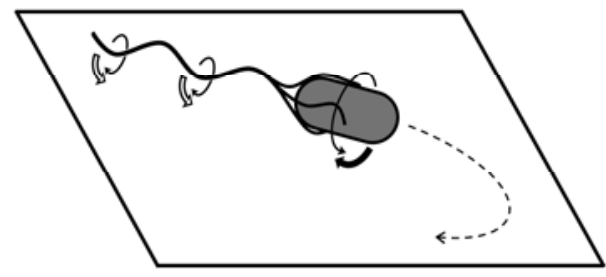

图 3 大肠杆菌接近下方固相表面时的游泳行为

$\mathrm{A}$ : 平滑泳动的大肠杆菌 HCB437 菌株在载玻片表面作圆周运动的 视频截图. 细胞旁数字显示图像记录时间. 细胞约倾斜 $14^{\circ}$, 并以约 $12 \mu \mathrm{m} / \mathrm{s}$ 的速度行进. 为了清楚显示细胞体的倾斜, 特意选择了一个 较长的细胞, 而且将显微镜聚焦到细胞体前端所在平面, 这样其后 端因散焦而模糊. 实时视频为网络版附录文件视频 S2, 记录速度为 $25 \mu \mathrm{s} /$ 幢; B: 倾角 $(\theta)$ 的流体动力学解析. 倾角的产生是由于两个不 同方向的扭矩(弯箭头)相互抵消. 其中一个扭矩(实心箭头)是由于 壁效应造成的细菌表面黏性曳力不均匀分布造成的; 另一个扭矩 (空心箭头) 是由细菌迎面液流产生的曳力造成的. 在达到临界角时, 细菌沿一个界面的平行面作零扭矩的位移; $\mathrm{C}$ : 圆周运动的流体动 力学解释. 由于固相界面存在而产生的、作用于细胞体的曳力, 与 细胞体的旋转(实心箭头)相拮抗, 从而产生一个右手螺旋方向的扭 矩; 而作用于鞭毛束下部的曳力, 与鞭毛的旋转(空心箭头)相拮抗, 从而产生一个左手螺旋方向的扭矩. 这两个扭矩的协调作用使细菌 沿圆环的路径泳动(虚箭头)

在培养试管中, 不具有趋化能力的平滑泳动大 肠杆菌突变体比野生型沉降速度快. 这是因为它们 无法通过转向后的向上泳动来消除重力的影响. 有 研究认为, 平滑泳动突变体细胞倾向于采取头朝下 的倾斜姿势 ${ }^{[56]}$. 他们认为, 这是重力造成的, 因为细
菌细胞体比细菌鞭毛束的沉降速度大得多. 假如这 种被动沉降真的存在，也只会在细菌处于远离底部 的位置起作用. 另外, 这种被动倾斜要求细菌细胞的 重心位置发生改变, 其可能性需要进一步探讨.

流体动力学相互作用会阻止细菌和底部界面产 生实际接触, 并使它们在一个与界面平行的空间平 面上保持平移 ${ }^{[6]}$. 在朝界面泳动时, 由于壁效应, 细 菌的速度会随着距离缩短而渐进式降低, 并最终在 某个距离处开始沿界面的平行方向前进 ${ }^{[57]}$. 实际观 测结果显示, 大肠杆菌通常在距离纯净石英表面大 约 $40 \mathrm{~nm}$ 处平行游动 ${ }^{[50]}$, 而且它们的游动速度会逐渐 降低到约 $16 \mu \mathrm{m} / \mathrm{s}^{[58]}$. 电脑模拟实验也显示, 细菌与 界面的距离会保持在纳米级别, 同时较长的细胞倾 向于在距离界面较远处泳动 ${ }^{[52]}$. 附录视频 S2 显示了 这一现象。

\section{3 近界面圆周运动}

在静止介质中, 大肠杆菌细胞在固相表面附 近泳动时会作圆周运动, 而且经常在转向后离开界 面 ${ }^{[58]}$. 流体动力学模拟显示, 作用于旋转中的细胞体 下部和鞭毛束下部的黏性曳力与作用于细胞体和 鞭毛束上部的曳力相比较大, 这是细菌产生圆周运动 的原因 ${ }^{[6,57]}$. 由于细胞体作顺时针旋转, 与之相拮抗 的壁曳力产生一个推动细胞体侧向偏移的扭矩; 而 沿逆时针方向旋转的鞭毛束的下部获得的黏性曳力 是朝相反方向的(图 3C). 在两个扭矩的共同作用下, 如果从细菌上方进行观察, 细胞体就会不断向右侧 滚动. 如果固相界面在上方, 就会看到细菌向左侧作 圆周运动 ${ }^{[59]}$.

Maeda 等人 ${ }^{[60]}$ 观察到, 细菌运动轨迹的弧半径 可以小到 $10 \mu \mathrm{m}$, 大到 $50 \mu \mathrm{m}$, 并认为半径的大小与 温度有关. 理论上讲, 圆周轨迹的半径等于细菌运动 速度除以面外转速(out-of plane rotation rate)的商 ${ }^{[57]}$. 而面外旋转速度由物体的物理尺度和它与壁之间的 距离决定 ${ }^{[6]}$. 细菌的细胞形态因而也会影响轨迹半径 的大小, 较长的细胞倾向于沿较大的弧线运动 ${ }^{[52]}$. 尽 管化感作用的细胞内信号传导网络对温度变化不敏 感 ${ }^{[61]}$, 用于驱动鞭毛旋转的质子动力势 (protonmotive force) 由细菌代谢产生, 随温度变化 ${ }^{[62]}$. 除细胞大小 和生长温度, 每个细胞的生理状态也会使泳动轨迹 的弧度更多变 ${ }^{[57]}$. 这是因为, 细菌可以通过细胞内二 
级信使一一环鸟苷二磷酸(cyclic di-GMP)对鞭毛旋转 的速度进行精确调控 ${ }^{[63,64]}$.

当大肠杆菌被限制在一个高度为几微米的浅槽 中, 浅槽的底部为琼脂糖, 其侧壁为氧化聚二甲基硅 氧烷(oxidized poly(dimethylsiloxane), PDMS), 它们 会沿着浅槽的右手边游动 ${ }^{[59]}$. 这是因为, 大肠杆菌倾 向于靠近多孔的琼脂糖游动, 尽量避开 PDMS 表面. 它们倾向于向右侧靠近是由于不同表面的差异动力 学特征造成对称破缺. 理论预期和实验结果都显示, 当通道的高度超过 $10 \mu \mathrm{m}$ 时, 向右侧游动的倾向消 失. 流体动力学相互作用只在分隔距离接近或者小 于物体的物理尺度时显著 ${ }^{[6]}$. 壁效应还造成大肠杆菌 在直径 $10 \mu \mathrm{m}$ 的毛细管中的趋化效率比在 $50 \mu \mathrm{m}$ 毛细 管中高很多. 这是因为, 它们在 $50 \mu \mathrm{m}$ 毛细管中不断 作圆周运动而无法有效前进 ${ }^{[65]}$. 综上所述, 可以推论 细菌在很细的毛细管中会沿螺旋状的路径前进, 因 为这时会有一个持续不断施加的壁效应存在. 它们 运动轨迹的螺距会随着毛细管孔径的缩小而增大.

单鞭毛细菌在固相界面附近也会作圆周运动(表 1). 这个现象在溶藻弧菌与非滑移表面的距离小于 $10 \mu \mathrm{m}$ 时非常显著 ${ }^{[66]}$. 可是, 它们作圆周运动时不是 在前进态, 而是在后退时 (图 4). 它们向前泳动时的 路径几乎是直的, 尽管速度随着相对于界面距离的 缩小而降低 ${ }^{[15,57]}$. 在游近界面时, 溶藻弧菌和新月柄 杆菌扩散胞所作的圆周运动方向相反. 这是由于它 们的鞭毛旋转方向相反 (表 1). 香茅醇假单胞杆菌 (Pseudomonas citronellolis) 和铜绿假单胞杆菌(Pseudomonas aeruginosa) 在靠近固相界面时也作圆周泳 动 (对于铜绿假单胞杆菌, 文献未标明方向 $)^{[23,25]}$. 研 究认为, 香茅醇假单胞杆菌是在前进态时作顺时针 圆周运动一一与大肠杆菌类似 ${ }^{[23]}$. 然而, 这样假设与 其他单鞭毛细菌的研究结果相矛盾. 如果假单胞杆 菌是在后退时作圆周运动, 那么假单胞杆菌和柄杆 菌的泳动方式是否一致?

需要指出, 在前面讨论的理论模型中, 细菌都被 简化为一个刚性的螺旋连接到一个球形的细胞上, 泳动时细胞的长轴和界面保持平行 ${ }^{[6,57]}$. 目前为止, 只有一种球形细菌细胞的游泳行为被简单研究过 ${ }^{[50]}$, 即椭圆的球形红细菌中偶尔出现的正球形个体. 它 们的确作圆周运动. 由于球形红细菌不会后退, 所以 它们一定是在前进时作圆周泳动的. 进一步研究其 他能动球形细菌的信号受体分布及其近壁泳动行为

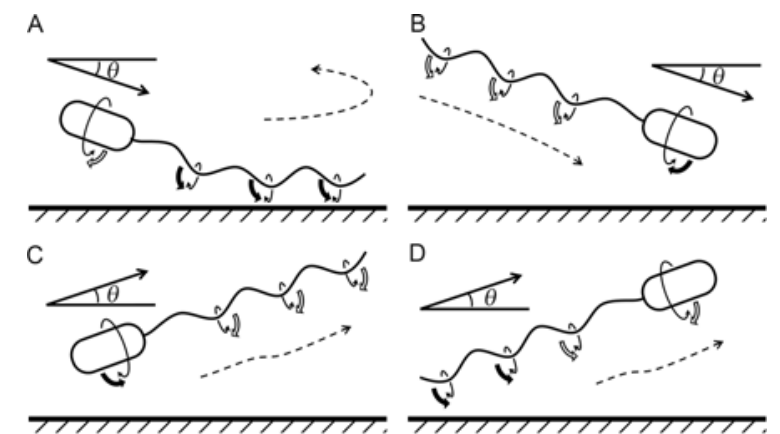

图 4 溶藻弧菌的近固相界面泳动行为

“ $\theta$ ”为细菌细胞纵轴与界面的角度. 虚箭头示潜在的游泳轨迹; 细 实心箭头示细胞体和鞭毛的旋转方向; 空心箭头示相拮抗的壁曳力 粗箭头示由壁电力和 DLVO 作用所产生的合力. A: 一个头朝下向 后退的细菌沿界面平移时的受力情况; $\mathrm{B}$ : 一个头朝下向前游动的 细菌沿界面平移时的相互作用力; C: 一个头朝下向后退的细菌在 界面附近的受力情况; $\mathrm{D}$ : 一个头朝上向前游动的细菌沿界面平移 时的受力情况

能够更加深入地揭示壁效应对趋化作用的影响.

一个杆状细菌以头朝下的姿势平移则需要构建 更加复杂的模型. Frymier 等人 ${ }^{[58]}$ 建议, 由 Derjaguin, Landan, Verwey 和 Overbeek 提出的胶体稳定性理论 (the theory of colloid stability, 简称 DLVO 理论)可以 用于解释大肠杆菌的圆周泳动问题. 根据 DLVO 理 论, 一个基于生物体表面和固相界面之间的静电斥 力(repulsive electrostatic force)和范德华引力 (attractive van der Waals force)的相互作用会在距离界面 4.5 $\mathrm{nm}$ 处形成一个次级吉布斯自由能最低区 (secondary Gibbs energy minimum) ${ }^{[67]}$. 这个次级最小自由能将 细胞稳定在距离界面一定距离处 ${ }^{[58]}$.

将壁效应和 DLVO 静电相互作用的理论结合起 来就可以解释单鞭毛细菌的圆周运动机理问题(图 4). 当细菌采取头朝上的姿势后退时, 细胞体和壁之间 的 DLVO 作用相对较弱, 而鞭毛和壁之间的相互作 用较强(图 4A). 作用于细胞体的壁电力与作用于鞭 毛的壁电力加上静电力合力的共同作用, 就会造成 细菌的圆周泳动. 当细菌采用其他的细胞取向时, 或者静电力主要施加于细胞体, 从而使整个细胞不 断侧滑 (图 4B 和 C), 或者瞬时作用于鞭毛, 使细菌加 速离开固相表面(图 4D). 有实验证据显示, 柄杆菌 扩散胞是在距离固相表面几百纳米范围内作圆周游 动 ${ }^{[22]}$, 正处于次级吉布斯自由能最低的区域.

当细菌后退时, 如果鞭毛向下倾斜, 那么其远端 就会被吉布斯最低自由能稳定. 在光学显微镜下可 
见, 溶藻弧菌的鞭毛在作圆周运动时中间会弯曲 ${ }^{[68]}$. 而且, DLVO 相互作用产生的扭矩会加速细菌运动. 溶藻弧菌的后退速度在界面附近比自由泳动时快 1.5 倍, 而向前泳动速度则不受界面存在的影响 ${ }^{[99]}$.

DLVO 相互作用可能在大肠杆菌泳动中起的作 用不大. 提高介质的离子强度可以加强 DLVO 相互 作用, 但是对大肠杆菌近界游泳行为没有影响 ${ }^{[70]}$. 大 肠杆菌的鞭毛是裸露的蛋白质丝, 而单鞭毛细菌的 鞭毛是有鞘的. 鞭毛鞘是细菌细胞外膜的延伸 ${ }^{[71]}$. 这 样的鞭毛与界面的 DLVO 相互作用比裸露的周生鞭 毛更强. 尽管改变载玻片表面疏水性能未能影响溶 藻弧菌的近界游泳行为 ${ }^{[66]}$, 改变介质离子强度可能 会有更深刻的影响.

微生物在趋氧作用(aerotaxis)和趋光作用(phototaxis) 中, 会经历液体和空气的界面. 这是一种自由 界面, 界面应力趋近于零. 有人认为, 这种情况下点 力 (point force)映像系统(image system)方向的改变会 造成细菌的逆时针圆周运动 ${ }^{[57]}$. 实验显示, 大肠杆菌 在含有 $0.002 \%$ Tween- 20 的缓冲液中沿逆时针方向作 圆周泳动 ${ }^{[72]}$. 在这个实验中还观察到, 细菌的长度与 泳动轨迹的半径成近乎线性相关. 需要指出, 此时的 界面并非完全自由界面, 液体表面分布着微小的表 面活性剂块斑. 在另一个类似的实验中, 大肠杆菌在 含有甘油的培养基中泳动. 靠近气液界面时, 顺时针 圆周运动和逆时针圆周运动都会发生 ${ }^{[73]}$. 溶藻弧菌 在含有 4-羟乙基哌嗪乙磺酸 [4-(2-hydroxyethyl)-1piperazineethanesulfonic acid, HEPES]的培养基与空气 的界面处也同时进行顺时针和逆时针两个方向的圆 周泳动, 而且这些圆周运动与是否前进或者后退无 关 ${ }^{[69]}$. 观测细菌在真正的完全自由界面的运动, 需要 全新的实验设计. 采用一种具有适当疏水表面的样 品槽, 从而有效降低无表面活性剂液滴的接触角 (contact angle), 并利用光声显微镜 (photoacoustic microscope $)^{[74]}$ 进行观测, 或许是解决问题的方法之 一, 因为光声显微镜可以比传统显微镜观测到更深 位置的目标.

\section{4 展望}

尽管此处讨论的成果多半是由物理学家完成的, 细菌的进化固然选择了特定的尺寸、形状及鞭毛形态
来适应物理规律. 这里只关注小型细菌, 完全是由于 对于大型细菌的实验观测和生物物理学模拟还非常 有限. 大型细菌通常生活在完全不同的生境, 所以也 有完全不同的行为. 例如, 双端生鞭毛的海洋弧菌 (长 $6 \mu \mathrm{m}$ ) 像红细菌(长 $2.5 \mu \mathrm{m}$ ) 一样沿细胞短轴方向运 动, 可它们却能够检测到自身细胞体两极的氧浓度 差异 ${ }^{[28,48]}$. 螺旋形的迂回螺菌(Spirillum volutans) 能够 长到 $140 \mu \mathrm{m}$ 长 $^{[75]}$. 它们通过同步转动每个细胞极上 的约 25 根鞭毛组成的鞭毛丛而游动. 鞭毛丛在游动 时看起来像是个模糊圆锥. 而且它们向细胞两极方 向游动的能力没有区别 ${ }^{[1]}$. 另一个小型的双端生鞭毛 细菌趋磁螺菌(Magnetospirillum magneticum AMB-1) (约 $3 \mu \mathrm{m}$ 长)也可以向细胞两级方向同样有效地游 泳 ${ }^{[76,77]}$. 比较这两种螺菌的游泳模式可以进一步了 解细胞大小对运动的影响.

另一个重要的问题是不同鞭毛形态的进化生物 学意义. 已知周生鞭毛细菌能够在半固相介质中或 者可湿固体表面进行另一种形式的运动一集群迁 移(swarming), 而具有端生单鞭毛的细菌, 除铜绿假 单胞杆菌外, 都不能集群迁移 ${ }^{[71,78]}$. 另外, 单鞭毛细 菌所特有的前进一后退机制使细菌能够在高剪切流环 境中流连在适宜区域; 而周生鞭毛细菌的前进-转向 机制则使它们在类似的环境中等同于无运动能力细 菌 ${ }^{[79]}$. 有些细菌具有两套鞭毛系统 ${ }^{[71]}$. 它们提供了 一个回答这个问题的理想系统. 同基因型的具有单 生鞭毛的个体和具有周生鞭毛的个体可以通过基因 敲除和重组制造出来, 并在完全相同的实验条件下 进行比较.

细菌细胞形态与运动能力必然存在相关性. 尽 管大多数生物物理学模拟都采用球形细胞完成, 而 且在自然界中的 218 个自由生存细菌属的能动种类 中, 有 $10 \%$ 是球菌 ${ }^{[80]}$, 目前为止, 既没有天然球菌, 也没有人造球菌的泳动行为被细致研究过. 细胞形 态的另一些方面, 如弧菌的手性方向问题(表 1), 还 远没有引起生物学家的注意. 在今天, 通过基因操 作, 可以很容易将弧菌拉直成杆菌 ${ }^{[19]}$, 或者将杆菌 弯成螺菌 ${ }^{[81]}$. 其他新实验技术的发展, 如显微制造 (microfabrication)使在严格控制条件下对单个细菌的 运动行为研究成为可能 ${ }^{[11]}$. 可以预见, 在不远的将 来, 分子生物学和生物物理学研究的结合将大大促 进人们对细菌运动机制的了解. 
感谢法国巴斯德研究所 Anthony P. Pugsley 和美国哈佛大学 Howard C. Berg 在写作过程中提供的宝贵意见、 法国 CNRS Joliot-Curie 实验室 Christophe Place 提供未发表实验结果及清华大学胡家信和美国麻省大学 Evan Palmer-Young 提供的帮助.

\section{参考文献}

1 Berg H C. E. coli in Motion. Biological and Medical Physics Biomedical Engineering. New York: Springer-Verlag, 2003

2 Wadhams G H, Armitage J P. Making sense of it all: bacterial chemotaxis. Nat Rev Mol Cell Biol, 2004, 5: 1024-1037

3 Blair D F. How bacteria sense and swim. Annu Rev Microbiol, 1995, 49: 489-522

4 Sourjik V, Armitage J P. Spatial organization in bacterial chemotaxis. EMBO J, 2010, 29: 2724-2733

5 Wei Y, Wang X, Liu J, et al. The population dynamics of bacteria in physically structured habitats and the adaptive virtue of random motility. Proc Natl Acad Sci USA, 2011, 108: 4047-4052

6 Ramia M, Tullock D L, Phanthien N. The role of hydrodynamic interaction in the locomotion of microorganisms. Biophys J, 1993, 65: $755-778$

7 Berg H C. Random Walks in Biology. Princeton: Princeton University Press, 1993

8 Dusenbery D B. Minimum size limit for useful locomotion by free-swimming microbes. Proc Natl Acad Sci USA, 1997, 94: 10949-10954

9 Berg H C, Brown D A. Chemotaxis in Escherichia coli analysed by three-dimensional tracking. Nature, 1972, 239: 500-504

10 Montesinos E. Change in size of Chromatium minus cells in relation to growth rate, sulfur content, and photosynthetic activity: a comparison of pure cultures and field populations. Appl Environ Microbiol, 1987, 53: 864-871

11 Ahmed T, Shimizu T S, Stocker R. Microfluidics for bacterial chemotaxis. Integr Biol, 2010, 2: 604-629

12 Mitchell J G, Martinez-Alonso M, Lalucat J, et al. Velocity changes, long runs, and reversals in the Chromatium minus swimming response. J Bacteriol, 1991, 173: 997-1003

13 Darnton N C, Turner L, Rojevsky S, et al. On torque and tumbling in swimming Escherichia coli. J Bacteriol, 2007, 189: 1756-1764

14 Macnab R M, Ornston M K. Normal-to-curly flagellar transitions and their role in bacterial tumbling. Stabilization of an alternative quaternary structure by mechanical force. J Mol Biol, 1977, 112: 1-30

15 Goto T, Nakata K, Baba K, et al. A fluid-dynamic interpretation of the asymmetric motion of singly flagellated bacteria swimming close to a boundary. Biophys J, 2005, 89: 3771-3779

16 Homma M, Oota H, Kojima S, et al. Chemotactic responses to an attractant and a repellent by the polar and lateral flagellar systems of Vibrio alginolyticus. Microbiology, 1996, 142: 2777-2783

17 Kudo S, Imai N, Nishitoba M, et al. Asymmetric swimming pattern of Vibrio alginolyticus cells with single polar flagella. FEMS Microbiol Lett, 2005, 242: 221-225

18 Magariyama Y, Masuda S Y, Takano Y, et al. Difference between forward and backward swimming speeds of the single polar-flagellated bacterium, Vibrio alginolyticus. FEMS Microbiol Lett, 2001, 205: 343-347

19 Cabeen M T, Charbon G, Vollmer W, et al. Bacterial cell curvature through mechanical control of cell growth. EMBO J, 2009, 28: $1208-1219$

20 Koyasu S, Shirakihara Y. Caulobacter crescentus flagellar filament has a right-handed helical form. J Mol Biol, 1984, 173: 125-130

21 Li G, Tang J X. Low flagellar motor torque and high swimming efficiency of Caulobacter crescentus swarmer cells. Biophys J, 2006, 91 : 2726-2734

22 Li G L, Tam L K, Tang J X. Amplified effect of Brownian motion in bacterial near-surface swimming. Proc Natl Acad Sci USA, 2008, 105: 18355-18359

23 Taylor B L, Koshland Jr D E. Reversal of flagellar rotation in monotrichous and peritrichous bacteria: generation of changes in direction. J Bacteriol, 1974, 119: 640-642

24 Vaituzis Z, Doetsch R N. Motility tracks: technique for quantitative study of bacterial movement. Appl Microbiol, 1969, 17: 584-588

25 Conrad J C, Gibiansky M L, Jin F, et al. Flagella and pili-mediated near-surface single-cell motility mechanisms in $P$. aeruginosa. Biophys J, 2011, 100: 1608-1616

26 Xie L, Altindal T, Chattopadhyay S, et al. Bacterial flagellum as a propeller and as a rudder for efficient chemotaxis. Proc Natl Acad Sci USA, 2011, 108: 2246-2251 
27 Stocker R. Reverse and flick: hybrid locomotion in bacteria. Proc Natl Acad Sci USA, 2011, 108: 2635-2636

28 Armitage J P, Macnab R M. Unidirectional, intermittent rotation of the flagellum of Rhodobacter sphaeroides. J Bacteriol, 1987, 169: $514-518$

29 Armitage J P, Schmitt R. Bacterial chemotaxis: Rhodobacter sphaeroide and Sinorhizobium meliloti-variations on a theme? Microbiology, 1997, 143: 3671-3682

30 Vladimirov N, Lebiedz D, Sourjik V. Predicted auxiliary navigation mechanism of peritrichously flagellated chemotactic bacteria. PLoS Comput Biol, 2010, 6: e1000717

31 Berg H C, Purcell E M. Physics of chemoreception. Biophys J, 1977, 20: 193-219

32 Alley M, Maddock J R, Shapiro L. Requirement of the carboxyl terminus of a bacterial chemoreceptor for its targeted proteolysis. Science, 1993, 259: 1754-1757

33 Maddock J R, Shapiro L. Polar location of the chemoreceptor complex in the Escherichia coli cell. Science, 1993, 259: 1717-1723

34 Ping L, Weiner B, Kleckner N. Tsr-GFP accumulates linearly with time at cell poles, and can be used to differentiate 'old' versus 'new' poles, in Escherichia coli. Mol Microbiol, 2008, 69: 1427-1438

35 Thiem S, Kentner D, Sourjik V. Positioning of chemosensory clusters in E. coli and its relation to cell division. EMBO J, 2007, 26: $1615-1623$

36 Greenfield D, McEvoy A L, Shroff H, et al. Self-organization of the Escherichia coli chemotaxis network imaged with super-resolution light microscopy. PLoS Biol, 2009, 7: e1000137

37 Sourjik V. Receptor clustering and signal processing in E. coli chemotaxis. Trends Microbiol, 2004, 12: 569-576

38 Boldog T, Grimme S, Li M, et al. Nanodiscs separate chemoreceptor oligomeric states and reveal their signaling properties. Proc Natl Acad Sci USA, 2006, 103: 11509-11514

39 Segall J E, Block S M, Berg H C. Temporal comparisons in bacterial chemotaxis. Proc Natl Acad Sci USA, 1986, 83: 8987-8991

40 Duke T A J, Bray D. Heightened sensitivity of a lattice of membrane receptors. Proc Natl Acad Sci USA, 1999, 96: 10104-10108

41 Sourjik V, Berg H C. Functional interactions between receptors in bacterial chemotaxis. Nature, 2004, 428: 437-441

42 Khursigara C M, Lan G, Neumann S, et al. Lateral density of receptor arrays in the membrane plane influences sensitivity of the $E$. coli chemotaxis response. EMBO J, 2011, 30: 1719-1729

43 Hansen C H, Sourjik V, Wingreen N S. A dynamic-signaling-team model for chemotaxis receptors in Escherichia coli. Proc Natl Acad Sci USA, 2010, 107: 17170-17175

44 Aquino G, Clausznitzer D, Tollis S, et al. Optimal receptor-cluster size determined by intrinsic and extrinsic noise. Phys Rev E, 2011, 83: 021914

45 Berg H C, Turner L. Cells of Escherichia coli swim either end forward. Proc Natl Acad Sci USA, 1995, 92: 477-479

46 Ping L. The asymmetric flagellar distribution and motility of Escherichia coli. J Mol Biol, 2010, 397: 906-916

47 Wadhams G H, Martin A C, Armitage J P. Identification and localization of a methyl-accepting chemotaxis protein in Rhodobacter sphaeroides. Mol Microbiol, 2000, 36: 1222-1233

48 Thar R, Kühl M. Bacteria are not too small for spatial sensing of chemical gradients: an experimental evidence. Proc Natl Acad Sci USA, 2003, 100: 5748-5753

49 Hill J, Kalkanci O, McMurry J L, et al. Hydrodynamic surface interactions enable Escherichia coli to seek efficient routes to swim upstream. Phys Rev Lett, 2007, 98: 068101

50 Vigeant M A S, Ford R M, Wagner M, et al. Reversible and irreversible adhesion of motile Escherichia coli cells analyzed by total internal reflection aqueous fluorescence microscopy. Appl Environ Microbiol, 2002, 68: 2794-2801

51 Hsu R, Ganatos P. The motion of a rigid body in viscous-fluid bounded by a plane Wall. J Fluid Mech, 1989, 207: 29-72

52 Shum H, Gaffney E A, Smith D J. Modelling bacterial behaviour close to a no-slip plane boundary: the influence of bacterial geometry. Philos Trans R Soc Lond A, 2010, 466: 1725-1748

53 Cabeen M T, Jacobs-Wagner C. The bacterial cytoskeleton. Annu Rev Genet, 2010, 44: 365-392

54 van den Ent F, Johnson C M, Persons L, et al. Bacterial actin MreB assembles in complex with cell shape protein RodZ. EMBO J, 2010, 29: $1081-1090$

55 Frankel R B, Bazylinski D A, Johnson M S, et al. Magneto-aerotaxis in marine coccoid bacteria. Biophys J, 1997, 73: 994-1000

56 Aswad D, Koshland Jr D E. Isolation, characterization and complementation of Salmonella typhimurium chemotaxis mutants. J Mol Biol, 1975, 97: 225-235

57 Lauga E, DiLuzio W R, Whitesides G M, et al. Swimming in circles: motion of bacteria near solid boundaries. Biophys J, 2006, 90: 
400-412

58 Frymier P D, Ford R M, Berg H C, et al. Three-dimensional tracking of motile bacteria near a solid planar surface. Proc Natl Acad Sci USA, 1995, 92: 6195-6199

59 DiLuzio W R, Turner L, Mayer M, et al. Escherichia coli swim on the right-hand side. Nature, 2005, 435: 1271-1274

60 Maeda K, Imae Y, Shioi J I, et al. Effect of temperature on motility and chemotaxis of Escherichia coli. J Bacteriol, 1976, 127: 1039-1046

61 Oleksiuk O, Jakovljevic V, Vladimirov N, et al. Thermal robustness of signaling in bacterial chemotaxis. Cell, 2011, 145: 312-321

62 Lowe G, Meister M, Berg H C. Rapid rotation of flagellar bundles in swimming bacteria. Nature, 1987, 325: 637-640

63 Boehm A, Kaiser M, Li H, et al. Second messenger-mediated adjustment of bacterial swimming velocity. Cell, 2010, 141: 107-116

64 Paul K, Nieto V, Carlquist W C, et al. The c-di-GMP binding protein YcgR controls flagellar motor direction and speed to affect chemotaxis by a backstop brake mechanism. Mol Cell, 2010, 38: 128-139

65 Berg H C, Turner L. Chemotaxis of bacteria in glass-capillary arrays - Escherichia coli, motility, microchannel plate, and light-scattering. Biophys J, 1990, 58: 919-930

66 Magariyama Y, Ichiba M, Nakata K, et al. Difference in bacterial motion between forward and backward swimming caused by the wall effect. Biophys J, 2005, 88: 3648-3658

67 Loosdrecht M C M, Norde W, Lyklema J, et al. Hydrophobic and electrostatic parameters in bacterial adhesion. Aquat Sci, 1990, 52: 103-114

68 Magariyama Y, Sugiyama S, Muramoto K, et al. Simultaneous measurement of bacterial flagellar rotation rate and swimming speed. Biophys J, 1995, 69: 2154-2162

69 Nakai T, Kikuda M, Kuroda Y, et al. Speed, trajectory and increment in the number of cells of singly flagellated bacteria swimming close to boundaries. J Biomech Sci Eng, 2009, 4: 2-10

70 Vigeant M, Ford R. Interactions between motile Escherichia coli and glass in media with various ionic strengths, as observed with a three-dimensional-tracking microscope. Appl Environ Microbiol, 1997, 63: 3474-3479

71 McCarter L L. Polar flagellar motility of the Vibrionaceae. Microbiol Mol Biol Rev, 2001, 65: 445-462

72 Di Leonardo R, Dell'Arciprete D, Angelani L, et al. Swimming with an image. Phys Rev Lett, 2011, 106: 038101

73 Lemelle L, Palierne J-F, Chatre E, et al. Counter-clockwise circular motion of bacteria swimming at air/liquid interface. J Bacteriol, 2010, 192: 6307-6308

74 Zhang H F, Maslov K, Stoica G, et al. Functional photoacoustic microscopy for high-resolution and noninvasive in vivo imaging. Nat Biotech, 2006, 24: 848-851

75 Garrity G M, Brenner D J, Krieg N R, et al. The Proteobacteria. Bergey's Manual of Systematic Bacteriology. In: Garrity G M, Brenner D J, Krieg N R, eds. Vol. 2. New York: Springer-Verlag, 2005

76 Lefèvre C T, Song T, Yonnet J P, et al. Characterization of bacterial magnetotactic behaviors by using a magnetospectrophotometry Assay. Appl Environ Microbiol, 2009, 75: 3835-3841

77 Matsunaga T, Sakaguchi T, Tadakoro F. Magnetite formation by a magnetic bacterium capable of growing aerobically. Appl Microbiol Biotechnol, 1991, 35: 651-655

78 Harshey R M. Bacterial motility on a surface: many ways to a common goal. Annu Rev Microbiol, 2003, 57: 249-273

79 Luchsinger R H, Bergersen B, Mitchell J G. Bacterial swimming strategies and turbulence. Biophys J, 1999, 77: 2377-2386

80 Young K D. The selective value of bacterial shape. Microbiol Mol Biol Rev, 2006, 70: 660-703

81 Weibel D B, DiLuzio W R, Whitesides G M. Microfabrication meets microbiology. Nat Rev Microbiol, 2007, 5: 209-218 